\title{
DUALITY IN FRACTIONAL MINIMAX PROGRAMMING
}

\author{
S. CHANDRA and VIVEKA KUMAR
}

(Received 27 March 1992; revised 7 September 1992)

\author{
Communicated by B. Mond
}

\begin{abstract}
Certain omissions in the recently introduced dual for fractional minimax programming problem 'minimize $\max _{y \in Y} f(x, y) / h(x, y)$, subject to $g(x) \leq 0$, are indicated and two modified duals for this problem are presented. Various fractional programming and generalized fractional programming duals are shown to be special cases of this study.
\end{abstract}

1991 Mathematics subject classification (Amer. Math. Soc.): $90 \mathrm{C} 32$.

\section{Introduction}

The study of minimax optimization problems has been of considerable interest in the past-see for example [7] and [8], and the references cited therein. Schmitendrof [14] considered a very general class of static minimax problems and presented necessary/sufficient optimality conditions for the same. Later, Tanimoto [15] constructed a dual to the problem studied by Schmitendrof [14] and proved various duality theorems under convexity assumptions on objective and constraint functions. Recently, Yadav and Mukherjee [16] attempted to construct a dual to the fractional analogue of the problem considered by Tanimoto [15] and presented a duality theorem similar to the one given in [15].

The purpose of this paper is to point out certain omissions and inconsistencies in the work reported in [16] and to present two modified models of the dual for the fractional minimax problem considered there. These models have been motivated by those due to Mond and Weir [11] for scalar fractional programming and Bector et alia [2] for generalized fractional programming problems.

This paper has been divided into four sections. Section 2 includes preliminaries

(C) 1995 Australian Mathematical Society 0263-6115/95 \$A2.00+0.00 
and statement of the problem, while Section 3 is devoted to the construction of the modified duals and establishing main duality results. Certain special cases are discussed in Section 4. For the sake of convenience and ready reference, we have followed the notations of [16] as far as possible.

\section{Problem Formulation and Preliminaries}

Let $R^{n}$ denote the $n$-dimensional Euclidean space and $R_{+}^{n}$ its non-negative orthant. Let $f(.,$.$) and h(.,$.$) be real valued differentiable functions on R^{n} \times R^{m}$ and $g: R^{n} \rightarrow R^{p}$ be convex and differentiable. Let $Y \subset R^{m}$ be compact and $X=\left\{x \in R^{n}: g(x) \leq 0\right\}$ with $f(x, y) \geq 0$ and $h(x, y)>0$ on $X$ for each $y \in Y$. Let $J=\{1,2, \ldots, p\}$ and $x \in X, \quad J(x)=\left\{j \in J: g_{j}(x)=0\right\}$ and $Y(x)=\left\{y \in Y: f(x, y) / h(x, y)=\sup _{z \in Y} f(x, z) / h(x, z)\right\}$.

Recently, Yadav and Mukherjee [16] considered the following problem (P):

$$
\min _{x \in X} \sup _{y \in Y}\{f(x, y) / h(x, y)\} \text {. }
$$

Let $K$ be the set of triplets $(s, t, y)$ where $s$ ranges over the positive integers such that $1 \leq s \leq n+1, t=\left(t_{1}, \ldots, t_{s}\right)$ an $s$-dimensional vector with $t \geq 0, \sum_{i=1}^{s} t_{i}=1$, $y=\left(y_{1}, \ldots, y_{s}\right)$ an $m s$-dimensional vector with $y_{i} \in Y(x)(i=1,2, \ldots, s)$ for some $x \in R^{n}$. Also for $x \in X, \quad(s, t, y) \in K, \quad y_{i} \in Y(x), \quad v=f\left(x, y_{i}\right) / h\left(x, y_{i}\right)$ we defined $H(s, t, y)=\left\{(x, \mu) \in R^{n} \times R^{p}: x\right.$ and $\mu$ satisfy $\left\{y_{1}, y_{2}, \ldots, y_{s}\right\} \subset Y(x)$, $\mu \in R_{+}^{p}$ and $\sum_{i=1}^{s}\left(t_{i} / h\left(x, y_{i}\right)\left(\nabla_{x} f\left(x, y_{i}\right)-v \nabla_{x} h\left(x, y_{i}\right)\right)+\sum_{j=1}^{p} \mu_{j} g_{j}(x)=0\right\}$.

With notation defined as above, Yadav and Mukherjee [16] introduced the dual (D) to $(\mathrm{P})$ as follows:

$$
\max _{(s, t, y) \in K} \sup _{(x, \mu) \in H(s, t, y)} F(x)+\sum_{j=1}^{p} \mu_{j} g_{j}(x),
$$

where $F(x)=\sup _{y \in Y} f(x, y) / h(x, y)$, and proved duality theorems under convexity assumptions on the objective and constraint functions. However, a closer look at the dual problem (D) suggests certain omissions and inconsistencies in its formulation which causes difficulties in the proof of Theorem 2.1 of [16]. For this, let us take $Y$ as a singleton. The problem (P) then becomes the standard fractional programming problem:

$$
\min _{x \in X} f(x) / h(x)
$$

while (D) reduces to,

$$
\max _{(x, \mu)}\left\{f(x) / h(x)+\sum_{j=1}^{p} \mu_{j} g_{j}(x)\right\}
$$


subject to $\nabla\left(f(x) / h(x)+\sum_{j=1}^{p} \mu_{j} g_{j}(x)\right)=0, \mu \geq 0$.

But it is well known (see for example Schaible [12]) that the above pair of primal and dual problems gives rise to a duality gap.

Examining the construction of the dual (D) one notes that in the definition of $H(s, t, y), v$ should also be treated as a variable, but then equation (2) as given in the proof of Theorem 2.1 of [16] does not hold.

In the following we shall correct these omissions by presenting two modified models of the dual to $(\mathrm{P})$ and establishing duality theorems with respect to each.

We shall use the following theorem and its corollary proved by Schmitendrof [14] for the following minimax problem:

$$
\min _{x \in X} \sup _{y \in Y} \phi(x, y)
$$

where $\phi(.,):. R^{n} \times R^{m} \rightarrow R$ is differentiable and $Y$ and $X$ are the same as stated for the problem $(\mathrm{P})$.

THEOREM 2.1. Let $x^{*}$ be a solution to the minimax problem $(\overline{\mathrm{P}})$. Then there exists a positive integer $\alpha$, scalars $\lambda_{i} \geq 0, i=1, \ldots, \alpha$, scalars $\mu_{i} \geq 0, i=1,2, \ldots, p$, vectors $y_{i} \in Y\left(x^{*}\right) i=1, \ldots, \alpha$, such that

$$
\begin{gathered}
\sum_{i=1}^{\alpha} \lambda_{i} \nabla \phi\left(x^{*}, y_{i}\right)+\sum_{j=1}^{p} \mu_{j} \nabla g_{j}\left(x^{*}\right)=0, \\
\mu_{j} g_{j}\left(x^{*}\right)=0, \quad j=1,2, \ldots, p, \\
\sum_{i=1}^{\alpha} \lambda_{i}+\sum_{j=1}^{p} \mu_{j} \neq 0 .
\end{gathered}
$$

Moreover, if $\beta$ is the number of non-zero $\mu_{j}$, then $1 \leq \alpha+\beta \leq n+1$.

COROLlaRY 2.1. If the vectors $\nabla g_{j}\left(x^{*}\right), j \in J\left(x^{*}\right)$ are linearly independent, then $\sum_{i=1}^{\alpha} \lambda_{i} \neq 0$ can replace $(1)$.

\section{Duality}

Let $f(., y)$ and $-h(., y)$ be convex functions of $x$ for every $y$ and $K$ be the set as defined in Section 2 . We now present two modified models of the dual to the minimax problem (P). 
Model I. Let $H_{1}(s, t, y)$ denote the set of all triplets $(x, \mu, v) \in R^{n} \times R^{p} \times R$ satisfying

$$
\sum_{i=1}^{s} t_{i} \nabla\left(f\left(x, y_{i}\right)-v h\left(x, y_{i}\right)\right)+\nabla\left(\sum_{j=1}^{p} \mu_{j} g_{j}(x)\right)=0
$$

$$
\sum_{i=1}^{s} t_{i}\left(f\left(x, y_{i}\right)-v h\left(x, y_{i}\right)\right) \geq 0
$$

$$
\begin{gathered}
\sum_{j=1}^{p} \mu_{j} g_{j}(x) \geq 0, \\
\left\{y_{1}, y_{2}, \ldots, y_{s}\right\} \in Y(x), \\
\sum_{i=1}^{s} t_{i}=1, \quad t_{i} \geq 0 \quad(i=1,2, \ldots, s), \quad \mu \geq 0 .
\end{gathered}
$$

In this model, we introduce a dual (D1) to the minimax problem (P) as follows:

$$
\max _{(s, t, y) \in K} \sup _{(x, \mu, v) \in H_{1}(s, t, y)} v
$$

If for a triplet $(s, t, y)$ in $K$ the set $H_{1}(s, t, y)$ is empty, then we define the supremum over it to be $-\infty$. The theorem given below establishes the duality relationship between problem (P) and (D1).

THEOREM 3.1. Let $x^{*}$ be an optimal solution of the problem (P) and let $\nabla g_{j}(x)$, $j \in J\left(x^{*}\right)$ be linearly independent. Then there exist $\left(s^{*}, t^{*}, \bar{y}\right) \in K, v^{*} \in R$ and $\mu^{*} \in R_{+}^{p}$ with $\left(x^{*}, \mu^{*}, v^{*}\right) \in H_{1}\left(s^{*}, t^{*}, \bar{y}\right)$ such that $\left(s^{*}, t^{*}, \bar{y}\right)$ and $\left(x^{*}, \mu^{*}, v^{*}\right)$ give an optimal solution to the problem (D1). Also, the two problems (P) and (D1) have the same extremal values.

PROOF. Since $x^{*}$ is an optimal solution of $(\mathrm{P})$ and $\nabla g_{j}\left(x^{*}\right), j \in J\left(x^{*}\right)$ are linearly independent, then by Theorem 2.1 and its corollary, there exists a positive integer $s^{*}$, $1 \leq s^{*} \leq n+1, t^{\circ}=\left(t_{1}^{\circ}, t_{2}^{\circ}, \ldots, t_{s}^{\circ}\right)$ with $t_{i}^{\circ} \geq 0\left(i=1, \ldots, s^{*}\right) \bar{y}=\left(\bar{y}_{1}, \ldots, \bar{y}_{s^{*}}\right)$ with $\bar{y}_{i} \in Y\left(x^{*}\right) \quad\left(i=1,2, \ldots, s^{*}\right)$ and $\mu^{\circ}=\left(\mu_{1}^{\circ}, \ldots, \mu_{p}^{\circ}\right) \in R_{+}^{p}$ such that

$$
\sum_{i=1}^{s^{*}} t_{i}^{\circ} \nabla\left(f\left(x^{*}, \bar{y}_{i}\right) / h\left(x^{*}, \bar{y}_{i}\right)\right)+\sum_{j=1}^{p} \mu_{j}^{\circ} \nabla g_{j}\left(x^{*}\right)=0,
$$

and

$$
\nabla\left(\sum_{j=1}^{p} \mu_{j} g_{j}\left(x^{*}\right)\right)=0
$$


Now defining $v^{*}=f\left(x^{*}, \bar{y}_{i}\right) / h\left(x^{*}, \bar{y}_{i}\right)$, Equation (7) gives,

$$
\sum_{i=1}^{s^{*}} t_{i}^{\circ} / h\left(x^{*}, \bar{y}_{i}\right)\left\{\nabla f\left(x^{*}, \bar{y}_{i}\right)-v^{*} \nabla h\left(x^{*}, \bar{y}_{i}\right)\right\}+\left(\sum_{j=1}^{p} \mu_{j}^{\circ} g_{j}\left(x^{*}\right)\right)=0 .
$$

Furthermore, taking

$$
t_{i}^{*}=\frac{t_{i}^{\circ} / h\left(x^{*}, \bar{y}_{i}\right)}{\sum_{i=1}^{s^{*}}\left(t_{i}^{\circ} / h\left(x^{*}, \bar{y}_{i}\right)\right)} \quad \text { and } \quad \mu^{*}=\frac{\mu^{\circ}}{\sum_{i=1}^{s^{*}}\left(t_{i}^{\circ} / h\left(x^{*}, \bar{y}_{i}\right)\right)}
$$

we have

$$
\sum_{i=1}^{s^{*}} t_{i}^{*}\left\{\nabla f\left(x^{*}, \bar{y}_{i}\right)-v^{*} \nabla h\left(x^{*}, \bar{y}_{i}\right)\right\}+\nabla \sum_{j=1}^{p} \mu_{j}^{*} g_{j}\left(x^{*}\right)=0,
$$

where $\mu^{*} \in R_{+}^{P}, t_{i}^{*} \geq 0$ for all $i$ and $\sum_{i=1}^{s^{*}} t_{i}^{*}=1$. We also have

$$
\sum_{i=1}^{s^{*}} t_{i}^{*}\left\{f\left(x^{*}, \bar{y}_{i}\right)-v^{*} h\left(x^{*}, \bar{y}_{i}\right)\right\}=0 \text { and } \sum_{j=1}^{p} \mu_{j}^{*} g_{j}\left(x^{*}\right)=0 .
$$

Hence $\left(x^{*}, \mu^{*}, v^{*}\right) \in H_{1}\left(s^{*}, t^{*}, \bar{y}\right)$.

We now show that $\left(x^{*}, \mu^{*}, v^{*}\right)$ attains the maximum of the following problem:

$\max v, \quad$ subject to $(x, \mu, v) \in H_{1}\left(s^{*}, t^{*}, \bar{y}\right)$.

For this, let $(x, \mu, v) \in H_{1}\left(s^{*}, t^{*}, \bar{y}\right)$. Using $\left\{\bar{y}_{1}, \bar{y}_{2}, \ldots, \bar{y}_{s}\right\} \subset Y\left(x^{*}\right)$ we get

$$
\begin{aligned}
v^{*}-v= & \left(\sum_{i=1}^{s^{*}} t_{i}^{*} f\left(x^{*}, \bar{y}_{i}\right)\right) /\left(\sum_{i=1}^{s^{*}} t_{i}^{*} h\left(x^{*}, \bar{y}_{i}\right)\right)-v \\
= & {\left[\sum_{i=1}^{s^{*}} t_{i}^{*}\left(f\left(x^{*}, \bar{y}_{i}\right)-v h\left(x^{*}, \bar{y}_{i}\right)\right)\right] / \sum_{i=1}^{s^{*}} t_{i}^{*} h\left(x^{*}, \bar{y}_{i}\right) } \\
\geq & {\left[\left\{\sum_{i=1}^{s^{*}} t_{i}^{*}\left(\nabla f\left(x, \bar{y}_{i}\right)-v \nabla h\left(x, \bar{y}_{i}\right)\right)\right\}\left(x^{*}-x\right)-\sum_{j=1}^{p} \mu_{j} g_{j}(x)\right]\left(1 / \sum_{i=1}^{s^{*}} t_{i}^{*} h\left(x^{*}, \bar{y}_{i}\right)\right) } \\
\geq & \left(\sum_{i=1}^{s^{*}} t_{i}^{*} h\left(x^{*}, \bar{y}_{i}\right)\right)^{-1}\left[\left\{\sum_{i=1}^{s^{*}} \nabla t_{i}^{*}\left(f\left(x, \bar{y}_{i}\right)-v h\left(x, \bar{y}_{i}\right)\right)\right.\right. \\
& \left.\left.\quad+\sum_{j=1}^{p} \mu_{j} \nabla g_{j}(x)\right\}\left(x^{*}-x\right)-\sum_{j=1}^{p} \mu_{j} g_{j}\left(x^{*}\right)\right]
\end{aligned}
$$

$\geq 0 \quad$ (because of (2) and the fact that $x^{*} \in X$ and $\mu \geq 0$ ). 
To complete the proof, we must show that for any $(s, t, y) \in K$,

$$
\sup _{(x, \mu, v) \in H_{1}(s, t, y)} \leq v^{*} .
$$

We may assume that $H_{1}(s, t, y)$ is non-empty. Now for any $(x, \mu, v) \in H_{1}(s, t, y)$, we have

$$
v=f\left(x, y_{i}\right) / h\left(x, y_{i}\right)=\sum_{i=1}^{s} t_{i} f\left(x, y_{i}\right) / \sum_{i=1}^{s} t_{i} h\left(x, y_{i}\right) .
$$

Also by (3), $v^{*} \geq \sum_{i=1}^{s} t_{i} f\left(x^{*}, y_{i}\right) / \sum_{i=1}^{s} t_{i} h\left(x^{*}, y_{i}\right)$.

Therefore

$$
\begin{aligned}
v^{*}-v \geq & \left(\sum_{i=1}^{s} t_{i} f\left(x^{*}, y_{i}\right) / \sum_{i=1}^{s} t_{i} h\left(x^{*}, y_{i}\right)\right)-v \\
= & \left(\sum_{i=1}^{s} t_{i} h\left(x^{*}, y_{i}\right)\right)^{-1}\left[\sum_{i=1}^{s} t_{i}\left(f\left(x^{*}, y_{i}\right)-v h\left(x^{*}, y_{i}\right)\right)\right] \\
\geq & {\left[\left\{\sum_{i=1}^{s} t_{i}\left(\nabla f\left(x, y_{i}\right)-v \nabla h\left(x, y_{i}\right)\right)+\sum_{j=1}^{p} \mu_{j} \nabla g_{j}(x)\right\}\left(x^{*}-x\right)-\sum_{j=1}^{p} \mu_{j} g_{j}\left(x^{*}\right)\right] } \\
& \cdot\left(\sum_{i=1}^{s} t_{i} h\left(x^{*}, y_{i}\right)\right)^{-1},
\end{aligned}
$$

which in view of convexity of $f,-h$ and $g$, is $\geq 0$.

The theorem follows.

MODEL II. Let us define $H_{2}(s, t, y)$ to be the set of all tuples $(x, \mu) \in R^{n} \times R^{p}$ satisfying

$$
\begin{gathered}
\nabla\left(\left(\sum_{i=1}^{s} t_{i} f\left(x, y_{i}\right)+\sum_{j=1}^{p} \mu_{j} g_{j}(x)\right) / \sum_{i=1}^{s} t_{i} h\left(x, y_{i}\right)\right)=0, \\
\sum_{i=1}^{s} t_{i} f\left(x, y_{i}\right)+\sum_{j=1}^{p} \mu_{j} g_{j}(x) \geq 0 \\
\left\{y_{1}, y_{2}, \ldots, y_{s}\right\} \in Y(x), \\
\mu \geq 0, \quad \sum_{i=1}^{s} t_{i}=1, \quad t_{i} \geq 0, \quad \forall i .
\end{gathered}
$$

The second modified form of the dual to $(\mathrm{P})$ is given as follows

$$
\max _{(s, t, y) \in K} \sup _{(x, \mu) \in H_{2}(s, t, y)}\left(\sum_{i=1}^{s} t_{i} f\left(x, y_{i}\right)+\sum_{j=1}^{p} \mu_{j} g_{j}(x)\right) / \sum_{i=1}^{s} t_{i} h\left(x, y_{i}\right) .
$$


As for Model I, if for a triplet $(s, t, y) \in K$ the set $H_{2}(s, t, y)$ is empty we define the supremum over it to be $-\infty$. We now present an analogous form of Theorem 3.1 , which establishes the duality relationship between the problems (P) and (D2).

THEOREM 3.2. Let $x^{*}$ be an optimal solution of the problem $(\mathrm{P})$ and let $\nabla g_{j}\left(x^{*}\right)$, $j \in J\left(x^{*}\right)$ be linearly independent. Then there exists $\left(s^{*}, t^{*}, \bar{y}\right)$ in $K$ and $\mu^{*} \in R_{+}^{P}$ with $\left(x^{*}, \mu^{*}\right) \in H_{2}\left(s^{*}, t^{*}, \bar{y}\right)$ such that $\left(s^{*}, t^{*}, \bar{y}\right)$ and $\left(x^{*}, \mu^{*}\right)$ give an optimal solution to problem (D2). The two problems (P) and (D2) have the same extremal values.

PROOF. Since $x^{*}$ is an optimal solution of $(\mathrm{P})$ and $\nabla g_{j}\left(x^{*}\right), j \in J\left(x^{*}\right)$ are linearly independent, there exists a positive integer $s^{*}, 1 \leq s^{*} \leq n+1, t^{\circ}=\left(t_{1}^{\circ}, t_{2}^{\circ}, \ldots, t_{s}^{\circ}\right)$ with $t_{i}^{\circ} \geq 0 \quad\left(i=1, \ldots, s^{*}\right), \bar{y}=\left(\bar{y}_{1}, \bar{y}_{2}, \ldots, \bar{y}_{s^{*}}\right)$ with $\bar{y}_{i} \in Y\left(x^{*}\right) \quad\left(i=1, \ldots, s^{*}\right)$ and $\mu^{\circ}=\left(\mu_{1}^{\circ}, \ldots, \mu_{p}^{\circ}\right) \in R_{+}^{p}$ such that

$$
\sum_{i=1}^{s^{\bullet}} t_{i}^{\circ} \nabla \frac{f\left(x^{*}, \bar{y}_{i}\right)}{h\left(x^{*}, \bar{y}_{i}\right)}+\sum_{j=1}^{p} \mu_{j}^{\circ} \nabla g_{j}\left(x^{*}\right)=0
$$

and

$$
\sum_{j=1}^{p} \mu_{j}^{\circ} g_{j}\left(x^{*}\right)=0
$$

But equation (13) implies

$$
\begin{aligned}
& \sum_{i=1}^{s^{*}}\left(\frac{t_{1}^{\circ}}{h\left(x^{*}, \bar{y}_{i}\right)}\right)\left[\nabla f\left(x^{*}, \bar{y}_{i}\right)-\left(f\left(x^{*}, \bar{y}_{i}\right) / h\left(x^{*}, \bar{y}_{i}\right)\right) \nabla h\left(x^{*}, \bar{y}_{i}\right)\right] \\
& \quad+\sum_{j=1}^{p} \mu_{j}^{\circ} \nabla g_{j}\left(x^{*}\right)=0
\end{aligned}
$$

and therefore by defining

$$
\begin{gathered}
t_{i}^{*}=\left(t_{i}^{\circ} / h\left(x^{*}, \bar{y}_{i}\right)\right) / \sum_{i=1}^{s^{*}}\left(t_{i}^{\circ} / h\left(x^{*}, \bar{y}_{i}\right)\right) \quad \text { and } \\
\mu^{*}=\mu^{\circ} / \sum_{i=1}^{s}\left(t_{i}^{\circ} / h\left(x^{*}, \bar{y}_{i}\right)\right)
\end{gathered}
$$

we obtain $\mu^{*} \in R_{+}^{p}, t_{i}^{*} \geq 0$ for all $i, \sum_{i=1}^{s^{*}} t_{i}^{*}=1$ and

$$
\begin{aligned}
\sum_{i=1}^{s^{*}} t_{i}^{*}\left(\nabla f\left(x^{*}, \bar{y}_{i}\right)-\right. & \left.\left(\sum_{i=1}^{s^{*}} t_{i}^{*} f\left(x^{*}, \bar{y}_{i}\right)\right)\left(\sum_{i=1}^{s^{*}} t_{i}^{*} h\left(x^{*}, \bar{y}_{i}\right)\right)^{-1} \nabla h\left(x^{*}, \bar{y}_{i}\right)\right) \\
+\sum_{j=1}^{p} \mu_{j}^{*} \nabla g_{j}\left(x^{*}\right) & =0 .
\end{aligned}
$$


On simplification this gives

$$
\nabla\left(\left(\sum_{i=1}^{s^{*}} t_{i}^{*} f\left(x^{*}, \bar{y}_{i}\right)+\sum_{j=1}^{p} \mu_{j}^{*} g_{j}\left(x^{*}\right)\right) / \sum_{i=1}^{s^{*}} t_{i}^{*} h\left(x^{*}, \bar{y}_{i}\right)\right)=0 .
$$

In view of (14),

$$
\sum_{i=1}^{s^{*}} t_{i}^{*} f\left(x^{*}, \bar{y}_{i}\right)+\sum_{j=1}^{p} \mu_{j}^{*} g_{j}\left(x^{*}\right) \geq 0 .
$$

Thus $\left(x^{*}, \mu^{*}\right) \in H_{2}\left(s^{*}, t^{*}, \bar{y}\right)$.

We now claim that $\left(x^{*}, \mu^{*}\right)$ is optimal for the following problem:

$\max _{(x, \mu)}\left(\sum_{i=1}^{s^{*}} t_{i}^{*} f\left(x, \bar{y}_{i}\right)+\sum_{j=1}^{p} \mu_{j} g_{j}(x)\right) / \sum_{i=1}^{s^{*}} t_{i}^{*} h\left(x^{*}, \bar{y}_{i}\right)$, subject to $(x, \mu) \in H_{2}\left(s^{*}, t^{*}, \bar{y}\right)$.

For any $(x, \mu) \in H_{2}\left(s^{*}, t^{*}, \bar{y}\right)$, we have

$$
\begin{aligned}
& \frac{\sum_{i=1}^{s^{*}} t_{i}^{*} f\left(x^{*}, \bar{y}_{i}\right)+\sum_{j=1}^{p} \mu_{j}^{*} g_{j}\left(x^{*}\right)}{\sum_{i=1}^{s^{*}} t_{i}^{*} h\left(x^{*}, \bar{y}_{i}\right)}-\frac{\sum_{i=1}^{s^{*}} t_{i}^{*} f\left(x, \bar{y}_{i}\right)+\sum_{j=1}^{p} \mu_{j} g_{j}(x)}{\sum_{i=1}^{s^{*}} t_{i}^{*} h\left(x, \bar{y}_{i}\right)} \\
& \geq \frac{\sum_{i=1}^{s^{*}} t_{i}^{*} f\left(x^{*}, \bar{y}_{i}\right)+\sum_{j=1}^{p} \mu_{j} g_{j}\left(x^{*}\right)}{\sum_{i=1}^{s^{*}} t_{i}^{*} h\left(x^{*}, \bar{y}_{i}\right)}-\frac{\sum_{i=1}^{s^{*}} t_{i}^{*} f\left(x, \bar{y}_{i}\right)+\sum_{j=1}^{p} \mu_{j} g_{j}(x)}{\sum_{i=1}^{s^{*}} t_{i}^{*} h\left(x, \bar{y}_{i}\right)} \\
& \geq \frac{\sum_{i=1}^{s^{*}} t_{i}^{*} h\left(x, \bar{y}_{i}\right)}{\sum_{i=1}^{s^{*}} t_{i}^{*} h\left(x^{*}, \bar{y}_{i}\right)}\left(x^{*}-x\right)^{T} \nabla\left(\frac{\sum_{i=1}^{s^{*}} t_{i}^{*} f\left(x, \bar{y}_{i}\right)+\sum_{j=1}^{p} \mu_{j} g_{j}(x)}{\sum_{i=1}^{s^{*}} t_{i}^{*} h\left(x, \bar{y}_{i}\right)}\right) \\
& \left.=0 \quad \text { (because }(x, \mu) \in H_{2}\left(s^{*}, t^{*}, \bar{y}\right)\right) .
\end{aligned}
$$

Hence we have proved the claim. To complete the proof we must show that for any $(s, t, y) \in K$,

$$
\begin{array}{r}
\sup _{(x, \mu) \in H_{2}(s, t, y)}\left(\sum_{i=1}^{s} t_{i} f\left(x, y_{i}\right)+\sum_{j=1}^{p} \mu_{j} g_{j}(x)\right) / \sum_{i=1}^{s} t_{i} h\left(x, y_{i}\right) \\
\leq\left(\sum_{i=1}^{s^{*}} t_{i}^{*} f\left(x^{*}, \bar{y}_{i}\right)+\sum_{j=1}^{p} \mu_{j}^{*} g_{j}\left(x^{*}\right)\right) / \sum_{i=1}^{s^{*}} t_{i}^{*} h\left(x^{*}, \bar{y}_{i}\right) .
\end{array}
$$


Now,

$$
\begin{aligned}
& \left(\sum_{i=1}^{s^{*}} t_{i}^{*} f\left(x^{*}, \bar{y}_{i}\right)+\sum_{j=1}^{p} \mu_{j}^{*} g_{j}\left(x^{*}\right)\right) / \sum_{i=1}^{s^{*}} t_{i}^{*} h\left(x^{*}, \bar{y}_{i}\right) \\
& \quad=\sum_{i=1}^{s^{*}} t_{i}^{*} f\left(x^{*}, \bar{y}_{i}\right) / \sum_{i=1}^{s^{*}} t_{i}^{*} h\left(x^{*}, \bar{y}_{i}\right) \\
& \quad \geq \sum_{i=1}^{s} t_{i} f\left(x^{*}, y_{i}\right) / \sum_{i=1}^{s} t_{i} h\left(x^{*}, y_{i}\right) \\
& \quad \geq\left(\sum_{i=1}^{s} t_{i} f\left(x^{*}, y_{i}\right)+\sum_{j=1}^{p} \mu_{j} g_{j}\left(x^{*}\right)\right) / \sum_{i=1}^{s} t_{i} h\left(x^{*}, y_{i}\right) .
\end{aligned}
$$

Thus

$$
\begin{aligned}
& \frac{\sum_{i=1}^{s^{*}} t_{i}^{*} f\left(x^{*}, \bar{y}_{i}\right)+\sum_{j=1}^{p} \mu_{j}^{*} g_{j}\left(x^{*}\right)}{\sum_{i=1}^{s^{*}} t_{i}^{*} h\left(x^{*}, \bar{y}_{i}\right)}-\frac{\sum_{i=1}^{s} t_{i} f\left(x, y_{i}\right)+\sum_{j=1}^{p} \mu_{j} g_{j}(x)}{\sum_{i=1}^{s} t_{i} h\left(x, y_{i}\right)} \\
& \geq \frac{\sum_{i=1}^{s} t_{i} f\left(x^{*}, y_{i}\right)+\sum_{j=1}^{p} \mu_{j} g_{j}\left(x^{*}\right)}{\sum_{i=1}^{s} t_{i} h\left(x^{*}, y_{i}\right)}-\frac{\sum_{i=1}^{s} t_{i} f\left(x, y_{i}\right)+\sum_{j=1}^{p} \mu_{j} g_{j}(x)}{\sum_{i=1}^{s} t_{i} h\left(x, y_{i}\right)} \\
& \geq \frac{\sum_{i=1}^{s} t_{i} h\left(x, y_{i}\right)}{\sum_{i=1}^{s} t_{i} h\left(x^{*}, y_{i}\right)}\left(x^{*}-x\right)^{T} \nabla\left(\frac{\sum_{i=1}^{s} t_{i} f\left(x, y_{i}\right)+\sum_{j=1}^{p} \mu_{j} g_{j}(x)}{\sum_{i=1}^{s} t_{i} h\left(x, y_{i}\right)}\right) \\
& \left.=0 \text { (because }(x, \mu) \in H_{2}(s, t, y)\right)
\end{aligned}
$$

and hence the first assertion of the theorem follows. It is clear that the optimal values of the primal and dual problem are the same.

REMARK 1. Modifications similar to ones introduced above are also required for the alternative duality theorem discussed in Section 3 of Yadav and Mukherjee [16].

\section{SPECIAL CASES}

(1) In case $Y$ is a singleton, the problem (P) becomes the standard fractional programming problem and duals (D1) and (D2) reduce to the well known duals of Schaible [12] and Bector [1] respectively. 
(2) For the case of generalized fractional programming ([2, 3, 4, 6 and 10]), the set $Y$ can be taken as the simplex $Y=\left\{y \in R^{m}: y_{i} \geq 0, \sum_{i=1}^{m} y_{i}=1\right\}$ and $f(x, y) / h(x, y)=\left(\sum_{i=1}^{m} y_{i} f_{i}(x) / \sum_{i=1}^{m} y_{i} h_{i}(x)\right)$. Then (D1) and (D2) reduce to the duals of [2] and [4]. Such problems in a much more abstract setting have been studied very recently by Gwinner and Jeyakumar in [9], where certain applications ([5 and 13]) have also been mentioned.

\section{ACKNOWLEDGEMENTS}

The authors wish to thank two referees for their valuable suggestions and citation of relevent literature including the recent reference [9].

\section{References}

[1] C. R. Bector, 'Duality in nonlinear fractional programming', Z. Oper. Res. 17 (1973), 183-193.

[2] C. R. Bector, S. Chandra and M. K. Bector, 'Generalized fractional programming duality: A parametric approach', J. Optim. Theory Appl. 60 (1988), 243-260.

[3] C. R. Bector and S. K. Suneja, 'Duality in nondifferentiable generalized fractional programming', Asia-Pacific J. Oper. Res. 5 (1988), 134-139.

[4] S. Chandra, B. D. Craven and B. Mond, 'Generalized fractional programming duality: A ratio game approach', J. Austral. Math. Soc. (Series B) 28 (1986), 170-180.

[5] B. D. Craven, Fractional programming (Heldermann, Berlin, 1988).

[6] J. P. Crouzeix, J. A. Ferland and S. Scheible, 'Duality in generalized fractional programming', Math. Prog. 27 (1983), 342-354.

[7] J. M. Danskin, 'The theory of max-min with applications', SIAM J.Appl. Math. 14(1966), 641-664.

[8] V. F. Demyanov and V. N. Malozemov, Introduction to minimax (Wiley, New York, 1974).

[9] J. Gwinner and V. Jeyakumar, 'A solvability theorem and minimax fractional programming', Applied Mathematics preprint AM91/7, University of New South Wales, 1991.

[10] R. Jagannathan and S. Schiable, 'Duality in generalized fractional programming via Farkas Lemma', J. Optim. Theory Appl. 41 (1983), 417-424.

[11] B. Mond and T. Weir, 'Generalized concavity and duality', in: Generalized concavity in optimization and economics, (eds. S. Schiable and W. T. Ziemba) (Academic Press, New York, 1981).

[12] S. Schiable, 'Fractional Programming I, Duality', Management Sci. 22 (1976), 858-867.

[13] S. Schiable and T. Ibaraki, 'Fractional Programming', European J. Oper. Res. 12 (1983), 325-338.

[14] W. E. Schmitendrof, 'Necessary conditions and sufficient conditions for static minimax problems', J. Math. Anal. Appl. 57 (1977), 683-693.

[15] S. Tanimoto, 'Duality for a class of nondifferentiable mathematical programming problems', $J$. Math. Anal. Appl. 79 (1981), 283-294.

[16] S. R. Yadav and R. N. Mukherjee, 'Duality for fractional minimax programming problems', $J$. Austral. Math. Soc. (Series B) 31 (1990), 484-492. 
Department of Mathematics

Indian Institute of Technology

Hauz Khas

New Delhi-110016

India 